\title{
NEMATÓIDE DAS LESÕES ASSOCIADO A MUDAS DE MANGUEIRA EM ASSU-RN ${ }^{1}$
}

\author{
GUSTAVO RUBENS DE CASTRO TORRES ${ }^{2 *}$; RUI SALES JÚNIOR ${ }^{3}$; LAUREEN MICHELLE HOULLOU ${ }^{2}$; \\ ANDREIA MITSA PAIVA NEGREIROS ${ }^{3}$
}

\begin{abstract}
RESUMO - O objetivo do presente trabalho foi relatar, pela primeira vez, população de Pratylenchus brachyurus associada a raízes de mangueira cv. Tommy Atkins no estádio de muda em solo naturalmente infestado no município de Assú, Rio Grande do Norte. A caracterização da referida população do nematóide foi fundamentada em estudo morfométrico realizado em 20 fêmeas coletadas das raízes. Os dados morfométricos foram comparados aos dados publicados por diferentes autores em trabalhos sobre a ocorrência da referida espécie de nematóide das lesões e permitiram a identificação da população como pertencente à $P$. brachyurus.
\end{abstract}

Palavras-chave: Pratylenchus brachyurus. Morfometria. Mangifera indica.

\section{ROOT LESION NEMATODE ASSOCIATED TO MANGO SEEDLING IN ASSU-RN}

\begin{abstract}
The aim of this study was to report for the first time Pratylenchus brachyurus population associated to mango cv. Tommy Atkins seedling roots, on naturally infested soil, on Assú municipality, Rio Grande do Norte State. The population of nematode was characterized based on a morphometric study that was done using 20 females collected from the roots. The morphometric data were compared to other data published by different authors in studies concerning to reporters of this species of root-lesion nematode, and it was possible to identify the population as P. brachyurus.
\end{abstract}

Keywords: Pratylenchus brachyurus. Morphometry. Mangifera indica.

\footnotetext{
*Autor para correspondência

${ }^{1}$ Recebido para publicação em 27/04/2015; aceito em 17/08/2015

${ }^{2}$ Centro de Tecnologias Estratégicas do Nordeste - CETENE - Laboratório de Pesquisas Aplicada à Biofábrica - LAPAB, Avenida Professor Luiz Freire, $\mathrm{n}^{\mathrm{o}}$ 1, Cidade Universitária, Recife (PE), CEP.: 50.740-540; gustavo.torres@cetene.gov.br, laureen.houllou@cetene.gov.

${ }^{3}$ Universidade Federal Rural do Semi Árido - UFERSA- Departamento de Ciências Vegetais, 59.625-900, Mossoró (RN); jrrui@hotmail.com,deia_mitsa@hotmail.com.
} 


\section{INTRODUÇ̃̃̃O}

O clima semiárido presente principalmente no sertão nordestino, com características de baixa umidade relativa do ar, baixo regime pluviométrico e elevada luminosidade, aproximadamente 3.500 horas de luz/ano, proporciona condições potenciais para o fortalecimento da fruticultura irrigada na região Nordeste do Brasil. Diante do citado cenário, alguns Estados nordestinos vêm se destacando como importantes produtores e exportadores de frutas no Brasil. O Rio Grande do Norte apresenta uma das três áreas frutícolas mais importantes do Nordeste, com destaque para a produção de frutas tropicais (IBGE, 2013).

De acordo com Torres et al. (2006), o núcleo de produção da fruticultura no Rio Grande do Norte situa-se no agropolo Assú/Mossoró, compreendendo os municípios de Mossoró, Assú, Upanema, Baraúna e Chapada do Apodi.

Os dados disponíveis sobre as exportações do Rio Grande do Norte demonstram que a manga (Mangifera indica L.), com uma área de produção no Estado de 2.704 ha (IBGE, 2013), ocupou o terceiro e quarto lugares entre as frutas mais exportadas pelo Rio Grande do Norte em 2012 e 2013, gerando rendas equivalentes a U\$ 9.018 e U\$ 6.568 milhões para os exportadores, respectivamente (FIERN, 2014).

Moura e Torres (2004) já assinalavam problemas enfrentados no Nordeste por produtores envolvidos com a fruticultura irrigada relacionados ao parasitismo de fitonematóides que se originaram por ocasião da instalação dos novos pomares em áreas infestadas, pelo uso comunitário de máquinas e equipamentos agrícolas e pela aquisição de mudas contaminadas.

Torres et al. (2004a; 2005a) relataram a ocorrência de Meloidogyne enterolobii Yang \& Eisenback (sin. Meloidogyne mayaguensis Ramamh \& Hirschmann) em goiabeira cv. 'Paluma' nos municípios de Touros (RN) e Limoeiro do Norte (CE) com mudas produzidas em viveiros em Petrolina (PE), respectivamente.

Um dos exemplos mais significativos de perdas que a aquisição de mudas contaminadas representou para a fruticultura nordestina nos últimos anos foi a redução ocorrida da área plantada com goiabeira (Psidium guajava L.) nos municípios de Pernambuco e Bahia, de 6.000 ha no ano de 2000 para 2.500 ha no ano de 2003, resultando na redução de mais de $50 \%$ da produção de goiaba, haja vista que a expansão dos plantios da referida mirtácea nesses Estados ocorreu, na maioria, com a utilização de mudas infectadas por M. enterolobii (CARNEIRO et al., 2006). Em 2009, Siqueira et al. citaram a detecção do referido parasita em goiabeira e mamoeiro (Carica papaya L.) no estado de Goiás, além de relatar sua presença associada a goiabeira nos estados do Rio de Janeiro, Rio Grande do Norte, Ceará, São Paulo, Paraná, Piauí, Espírito Santo, Paraíba, Mato
Grosso do Sul, Santa Catarina e Rio Grande do Sul, sendo provável que a referida disseminação pode ter sido favorecida pelo comércio de mudas utilizandose solo como substrato.

Exemplo de medida que visa impedir a disseminação de fitonematóides através de mudas produzidas utilizando-se solo como substrato foi adotada no estado do Rio de Janeiro com a publicação no DOE em 31 de março de 2015 pela Secretaria de Estado de Agricultura e Pecuária da Resolução SEAPEC n 69 de 26 de março de 2015, que classifica o nematóide do café (Coffea spp. L), Meloidogyne incógnita (Kofoid \& White) Chitwood, como praga prioritária e estabelece medidas para o controle da praga em cafezais e dá outras providências. Na referida Resolução consta no artigo quarto que fica proibida a produção e comercialização, no Estado do Rio de Janeiro, de mudas de cafeeiro produzidas com a utilização de solo como substrato, mesmo que para plantio na mesma região. Através do artigo quinto da citada Resolução fica também proibida a entrada de mudas de café no território fluminense vindas de outras Unidades da Federação com a utilização de solo como substrato (RIO DE JANEIRO, 2015).

De acordo com Goulart (2008), nematóides pertencentes ao gênero Pratylenchus são mundialmente reconhecidos como um dos maiores problemas em culturas de importância econômica como, por exemplo, soja (Glycine max L.), milho (Zea mays L.), feijoeiro (Phaseolus L. sp.), cana-deaçúcar (hídridos de Saccharum L. sp.), além de diversas forrageiras, hortaliças e fruteira, todas importantes para o Rio Grande do Norte.

Em estudos anteriores, Ferraz (1999) relacionou as seguintes espécies de Pratylenchus Filipjev relatadas no Brasil, quais sejam: $P$. brachyurus (Goodfrey) Filipjev e Schuurmans Stekhoven, $P$. coffeae (Zimmermann) Filipjev e Schuurmans Stekhoven, $P$. jordaniensis Hashim, P. neglectus (Rensch) Filipjev \& Stekhoven, P. penetrans (Cobb) Chitwood e Oteifa, P. pseudopratensis Seinhorst, $P$. scribneri Steiner, $P$. vulnus Allen e Jensen e $P$. zeae Grahan. Destas, foram relatadas em associação à mangueira, no Brasil, as espécies $P$. brachyurus e $P$. zeae.

Gonzaga (2006) relata que o gênero Pratylenchus foi alvo de sete revisões e que na última foram listadas 64 espécies válidas, sendo incluídas novas descrições: P. brzeskii sp. n. (KARSSEN; WAEYENBERGE; MOENS, 2000); P. arlingtoni sp. n. (HANDOO; CARTA; SKANTAR, 2001); $P$. jaehni sp. n. (INSERRA et al., 2001); P. gongjuensis sp. n. (CHOI et al., 2006); e P. dunensis sp. n. (PENA et al., 2006). O gênero passou a contar com 69 espécies válidas. Entretanto, o número de espécies consideradas é variável de acordo com diferentes autores (HANDOO et al., 2008). Mais recentemente, Carvalho (2013) destacou que o gênero Pratylenchus possui cerca de 70 espécies, das quais apenas seis são as mais frequentemente encontradas 
associadas a diferentes culturas, no Brasil, a saber: P. brachyurus; P. coffeae; P. jaehni; P. penetrans; P. vulnus; e P. zeae.

A taxonomia do gênero Pratylenchus tem sido tema de considerável número de publicações (CASTILLO; VOVLAS, 2007; MIZUKUBO et al., 2007; TROCCOLI et al., 2008; PALOMARESRIUS et al., 2010; LIRA, 2013; LIRA et al. 2014). Por meio de vários desses estudos foi demonstrado que medições dos caracteres-diagnósticos comprimento do estilete e valor $V \%$ (distância entre a cabeça e a vulva expressa em números percentuais do comprimento do corpo) eram variáveis taxonômicas menos afetadas por fatores bióticos e abióticos e provaram ser confiáveis como característicasdiagnósticas do gênero Pratylenchus (CASTILLO; VOVLAS, 2007).

Diante dessa premissa, o presente trabalho teve por objetivo identificar ao nível de espécie e caracterizar morfometricamente uma população de $P$. brachyurus obtida das raízes de mudas de mangueira cv Tommy Atkins cultivadas em solo naturalmente infestado no município de Assú (RN), assim como relatar a primeira ocorrência de população de $P$. brachyurus na citada cultura no Rio Grande do Norte.

\section{MATERIAL E MÉTODOS}

A presente ocorrência foi efetuada em outubro de 2004, quando foram enviadas ao laboratório de Fitonematologia da Universidade Federal Rural de Pernambuco - UFRPE, mudas de mangueira cultivar 'Tommy Atkins', produzidas em propriedade localizada no município de Assú com a finalidade de comercialização. A parte aérea das plantas apresentava sintomas de amarelecimento e declínio, e os sistemas radiculares tinham a maioria das raízes totalmente necrosadas e poucas viáveis. De acordo com o produtor, o solo utilizado para o preparo do substrato onde foram plantadas as mudas para comercialização foi proveniente de área ocupada com vegetação nativa da Caatinga, não tendo sido realizado tratamento prévio para desinfestação.

Em laboratório, quatro mudas plantadas em sacos plásticos contendo $2.000 \mathrm{~cm}^{3}$ de solo, proveniente da mesma área onde foram produzidas as mudas de mangueira, foram utilizadas isoladamente como repetições para análise nematológica. De cada muda, todo o solo proveniente da rizosfera foi destinado ao processamento de extração dos nematóides por flotação-centrífuga, e após a separação do solo os sistemas radiculares foram cortados, pesados e homogeneizados isoladamente, sendo retiradas de cada amostra alíquotas de 10 gramas de raízes para extração dos nematóides por meio de trituração em liquidificador, durante 20 segundos, associada à flotaçãocentrífuga (JENKINS, 1964).
As suspensões obtidas do processamento do solo e raízes de cada planta foram levadas ao microscópio óptico binocular (Nikon E200) para identificação dos fitonematóides incidentes ao nível de gênero de acordo com Mai e Mullin (1996) e determinação da densidade populacional. Os resultados foram computados em número de espécimes por $300 \mathrm{~cm}^{3}$ de solo e $10 \mathrm{~g}$ de raízes.

De cada suspensão proveniente das raízes, espécimes foram utilizados para confecção de lâminas semipermanentes, em água, visando a realização da classificação ao nível de espécie de acordo com a chave para classificação de espécies do gênero Pratylenchus segundo Handoo e Golden (1989) e do estudo morfométrico, mediante utilização de ocular micrométrica para medir as estruturas e dimensões dos nematóides, para caracterização da população em questão, tomando-se como base os valores médios e intervalos de confiança das variáveis: comprimento do corpo $(L)$, razão entre comprimento do corpo e largura máxima do corpo $(a)$, razão entre o comprimento do corpo e o comprimento do esôfago (b), razão entre o comprimento do corpo e o comprimento da cauda $(c)$, comprimento do estilete $(s)$ e posição relativa da vulva $(V \%)$. Os valores médios e intervalos de confiança foram obtidos a partir da medição das variáveis anteriormente citadas de 20 espécimes do sexo feminino, provenientes das raízes.

A morfologia dos espécimes analisados, os valores médios dos caracteres morfométricos e respectivos intervalos de confiança foram comparados aos dados descritos na chave de classificação de Handoo e Golden (1989) com o objetivo de determinar a espécie a qual a população em estudo pertencia bem como aos resultados obtidos em estudos morfométricos realizados por Thorne (1961), Roman e Hirschmann (1969) e Café Filho e Huang (1988), Handoo e Golden (1989) e Torres et al. (2004b) visando a caracterização morfométrica da referida população.

\section{RESULTADOS E DISCUSSÃO}

Nas suspensões provenientes do solo foi detectada uma média de 29 fêmeas adultas e juvenis em diferentes estádios de desenvolvimento e apenas um macho, pertencentes ao gênero Pratylenchus, em $300 \mathrm{~cm}^{3}$ de solo. Nas raízes foi encontrada uma média de 15 fêmeas e formas juvenis por 10 gramas.

Os valores dos intervalos de confiança (I.C.) das variáveis ' $L$ ', ' $a$ ', ' $b$ ', e 'c $c$ para a população objeto do presente estudo estiveram contidos nos I.C. das variáveis referentes às populações descritas em estudos realizados por outros autores e citados na Tabela 1, como também pertencentes à espécie P. brachyurus, com exceção dos I.C. encontrados para ' $a$ ' e ' $b$ ' e relatados por Torres et al. 2004b. 
Tabela 1. Mensurações* de fêmeas de Pratylenchus sp. associadas a raízes de mudas de mangueira cv. Tommy Atkins produzidas no município de Assú $(\mathrm{RN})$ e dados referentes a populações descritas por outros autores como pertencentes à espécie Pratylenchus brachyurus. Mossoró (RN), UFERSA, 2004.

\begin{tabular}{|c|c|c|c|c|c|}
\hline \multirow{2}{*}{ Espécie } & \multirow{2}{*}{ Referência** } & \multirow{2}{*}{$\mathrm{P}$} & \multicolumn{3}{|c|}{ Variáveis } \\
\hline & & & ${ }^{1} L(\mu m)$ & ${ }^{2} a$ & ${ }^{3} b$ \\
\hline \multirow{10}{*}{ P. brachyurus } & \multirow{2}{*}{ Thorne (1961) } & Média & - & - & - \\
\hline & & ${ }^{7} \mathrm{I} . \mathrm{C}$. & $450,00-750,00$ & $20,00-29,00$ & $5,60-6,10$ \\
\hline & \multirow{2}{*}{$\begin{array}{l}\text { Roman e Hirschmann } \\
\text { (1969) }\end{array}$} & Média & 567,81 & 22,83 & 6,09 \\
\hline & & I.C. & $398,60-656,00$ & $15,40-26,40$ & $5,50-7,00$ \\
\hline & \multirow{2}{*}{$\begin{array}{l}\text { Café Filho e Huang } \\
\text { (1988) }\end{array}$} & Média & 532,00 & 30,30 & 5,90 \\
\hline & & I.C. & $490,00-560,00$ & $28,00-35,00$ & $5,10-6,40$ \\
\hline & \multirow{2}{*}{$\begin{array}{l}\text { Handoo e Golden } \\
\text { (1989) }\end{array}$} & Média & - & - & - \\
\hline & & I.C. & $390,00-750,00$ & $15,00-29,00$ & $5,00-10,00$ \\
\hline & \multirow{2}{*}{$\begin{array}{l}\text { Torres et al. } \\
\text { (2004b) }\end{array}$} & Média & 570,00 & 16,85 & 9,59 \\
\hline & & I.C. & $540,00-600,00$ & $15,90-17,80$ & $7,80-11,30$ \\
\hline \multirow{2}{*}{ Pratylenchus sp. } & \multirow{2}{*}{ População Assú } & Média & 499,28 & 23,81 & 5,57 \\
\hline & & I.C. & $468,58-529,98$ & $22,70-24,92$ & $4,96-6,18$ \\
\hline
\end{tabular}

*Mensurações obtidas a partir de 20 fềmeas. ** Referências que apresentam dados morfométricos de populações de $P$. brachyurus estudadas em diferentes regiões geográficas. ${ }^{1} L=$ comprimento do corpo, ${ }^{2} a=$ razão entre o comprimento do corpo e a largura máxima do corpo, ${ }^{3} b=$ razão entre o comprimento do corpo e o comprimento do esôfago, ${ }^{4} c=$ razão entre o comprimento do corpo e o comprimento da cauda, ${ }^{5} \mathrm{~s}=$ comprimento do estilete, ${ }^{6} \mathrm{~V} \%=$ posição relativa da vulva. ${ }^{7}$ I.C. $=$ intervalo de confiança.

Tabela 1. Continuação

\begin{tabular}{|c|c|c|c|c|c|}
\hline \multirow{2}{*}{ Espécie } & \multirow{2}{*}{ Referência** } & \multirow{2}{*}{$\mathrm{P}$} & \multicolumn{3}{|c|}{ Variáveis } \\
\hline & & & ${ }^{4} \mathrm{c}$ & ${ }^{5} s(\mu \mathrm{m})$ & ${ }^{6} V^{\circ}$ \\
\hline \multirow{10}{*}{ P. brachyurus } & Thorne & Média & - & - & - \\
\hline & $(1961)$ & ${ }^{7} \mathrm{I} . \mathrm{C}$. & $17,00-21,00$ & $19,00-22,00$ & $83,00-89,00$ \\
\hline & Roman e Hirschmann & Média & 17,96 & 18,36 & 84,20 \\
\hline & (1969) & I.C. & $14,10-21,50$ & $17,40-19,20$ & $80,00-87,00$ \\
\hline & Café Filho e Huang & Média & 17,50 & 19,20 & 85,20 \\
\hline & $(1988)$ & I.C. & $15,00-21,00$ & $17,00-21,00$ & $83,00-87,00$ \\
\hline & Handoo e Golden & Média & - & - & - \\
\hline & (1989) & I.C. & $13,00-28,00$ & $17,00-22,00$ & $82,00-89,00$ \\
\hline & Torres et al. & Média & 18,03 & 85,10 & 16,62 \\
\hline & $(2004 a)$ & I.C. & $16,40-19,70$ & $15,30-17,90$ & $82,80-87,40$ \\
\hline \multirow{2}{*}{ Pratylenchus sp. } & & Média & 18,15 & 16,51 & 84,00 \\
\hline & Populaçao Assu & I.C. & $17,12-19,18$ & $16,09-16,93$ & $83,07-85,33$ \\
\hline
\end{tabular}

*Mensurações obtidas a partir de 20 fêmeas. ** Referências que apresentam dados morfométricos de populações de $P$. brachyurus estudadas em diferentes regiões geográficas. ${ }^{1} L=$ comprimento do corpo, ${ }^{2} a=$ razão entre o comprimento do corpo e a largura máxima do corpo, ${ }^{3} b=$ razão entre o comprimento do corpo e o comprimento do esôfago, ${ }^{4} c=$ razão entre o comprimento do corpo e o comprimento da cauda, ${ }^{5} s=$ comprimento do estilete, ${ }^{6} \mathrm{~V} \%=$ posição relativa da vulva. ${ }^{7} \mathrm{I} . \mathrm{C} .=$ intervalo de confiança.

Em relação a ' $s$ ' da população avaliada, o I.C. esteve contido apenas no I.C. da população assinalada pela primeira vez por Torres et al. (2004a), associada às raízes de meloeiro (Cucumis melo L.) no estado do Rio Grande do Norte, embora o limite máximo de ' $s$ ' para a população em estudo $(16,93 \mu \mathrm{m})$ tenha sido próximo aos limites mínimos da referida variável em relação às populações estudadas e identificadas como pertencentes à espécie $P$. brachyurus $(17,0 \mu \mathrm{m})$ nos estudos realizados por Café Filho e Huang (1988) e Handoo e Golden (1989) (Tabela 1).

$\mathrm{O}$ fato do valor de I.C. do comprimento do estilete ' $s$ ' encontrado no presente estudo não estar contido na maioria dos I.C. encontrados pelos demais autores não isenta a possibilidade da população de Assú pertencer à espécie $P$. brachyurus, tendo em vista Ferraz (1999) ressaltar que a dificuldade de visualizar com exatidão a extremidade apical do estilete, associada a grande proporção assumida por erros ocorrentes em mensurações de estruturas tão pequenas, é apontada como justificativa maior para as variações observadas em relação a essa variável. Segundo o referido autor, o comprimento do estilete tem importância limitada para identificação ao nível de espécie, pois as variações intraespecíficas são comuns. 
Quanto a $V \%$, o valor de I.C. encontrado para referida variável no presente estudo ficou contido em todos os intervalos de confiança citados pelos autores como sendo característico da espécie $P$. brachyurus (Tabela 1).

No presente estudo, a população avaliada apresentou apenas um macho em $300 \mathrm{~cm}^{3}$ de solo e ausência deste nas raízes da hospedeira, o que pode indicar que se trata de uma espécie de reprodução partenogenética a exemplo de $P$. zeae, $P$. brachyurus ou $P$. neglectus. Apesar da semelhança é válido ressaltar que o dado referente ao I.C. de $V \%$ obtido $(83,07-85,33)$ exclui a possibilidade dessa população pertencer a espécie $P$. zeae por não estar contido e ter se situado acima dos valores de I.C. encontrados pelos autores citados na Tabela 1, quais sejam: 68,00-76,00 (THORNE, 1961); 69,00-75,00 (ROMAN; HIRSCHMANN, 1969); 68,00-71,00 (CAFÉ FILHO; HUANG, 1988); e 66,00-76,00 (HANDOO; GOLDEN, 1989) como característicos para essa espécie. É válido considerar que conforme relato de P. brachyurus em meloeiro '(Cucumis melo L.) realizado por Torres et al. (2004b), a identificação ao nível de espécie no presente estudo além de ter sido confirmada com base nos critérios descritos na chave de classificação de Handoo e Golden (1989) fundamentou-se nas afirmações feitas por Ferraz (1999), que segundo o mesmo $V \%$ é uma variável útil na separação de espécies, sendo considerada como uma das mais seguras para fins de diagnóstico, como também por Castillo e Vovlas (2007), que citam ser a referida variável comprovadamente confiável como característica-diagnóstica do gênero Pratylenchus (CASTILLO; VOVLAS, 2007).

O presente relato chama a atenção dos produtores para o cuidado necessário com a sanidade do material propagativo adquirido visando a implantação de novos pomares, tendo em vista que, segundo Ferraz (1999), o gênero Pratylenchus, vulgarmente conhecido como nematóides das lesões radiculares, constitui o segundo grupo de fitonematóides mais importante para a agricultura, sendo causadores de galhas do gênero Meloidogyne Goeldi, como o primeiro em ordem de importância.

É importante citar que esse não é o primeiro relato da presença de mudas de fruteiras contaminadas com fitonematóides produzidas em viveiros em Assú (RN). Em estudos anteriores Torres et al. (2005b; 2007) detectaram a presença dos fitonematóides Hemicycliophora catarinenesis Costa Manso e $M$. enterolobii ( $M$. mayaguensis), respectivamente, associados a mudas de goiabeira cv. 'Paluma' comercializadas em viveiros em Assú (RN).

Vale salientar ainda que embora não seja errado especular a possibilidade da existência de populações nativas dos referidos parasitos em vegetação de Caatinga, a aquisição de mudas contaminadas pelos citados patógenos representa um dos fatores que pode oferecer riscos com níveis de alta gravidade financeira para a fruticultura irrigada por poder ocasionar prejuízos irreparáveis. Sendo assim, torna-se urgente a adoção de medidas sanitárias rígidas quanto a fiscalização e orientação técnica nos viveiros comerciais para que haja prevenção à disseminação dos fitonematóides.

\section{CONCLUSÃO}

Os dados resultantes da análise da morfologia e dos caracteres morfométricos obtidos permitiram identificar a população associada às raízes de mudas de mangueira cv. 'Tommy Atkins' como pertencente a espécie $P$. brachyurus, sendo o primeiro relato da espécie fitopatogênica em mangueira no município de Assú (RN).

\section{AGRADECIMENTOS}

Os autores agradecem ao Conselho Nacional de Desenvolvimento Científico e Tecnológico $(\mathrm{CNPq})$ e à FAPERN pela disponibilização de recursos que viabilizaram o desenvolvimento da presente pesquisa, bem como a bolsa de produtividade do segundo autor.

\section{REFERÊNCIAS}

CAFÉ FILHO, A. C.; HUANG, C. S. Nematóides do gênero Pratylenchus no Brasil. Fitopatologia Brasileira, Fortaleza, v. 13, n. 3, p. 232-235, 1988.

CARNEIRO, R. M. D. G. et al. Primeiro registro de Meloidogyne mayaguensis parasitando plantas de tomate e pimentão resistentes à meloidoginose no estado de São Paulo. Nematologia Brasileira, Brasília, v. 30, n. 1, p. 81-86, 2006.

CARVALHO, C. et al. Densidade populacional de Pratylenchus spp. em pastagens de Brachiaria spp. e sua influência na disponibilidade e na qualidade da forragem. Revista Ceres, Viçosa, v. 60, n. 1, p. 3037, 2013.

CASTILLO, P.; VOVLAS N. Pratylenchus (Nematoda, Pratylenchidae): diagnosis, biology, pathogenicity and management. Nematology Monographs and Perspectives, St. Paul, v. 6, 529 p. 2007.

CHOI, D. et al. A new and one unrecorded species of Pratylenchus from Korea (Nematoda: Pratylenchidae). Journal of Asia Pacific Entomology, Seul, v. 9, n. 1, p. 5-9, 2006

FIERN - FEDERAÇÃO DAS INDÚSTRIAS DO ESTADO DO RIO GRANDE DO NORTE. Dados 
Estatísticos: Exportações do RN - ano de 2013. Natal, 2013. Disponível em: $<$ http://www.fiern.org.br/ images/pdf/espaco_empresarial/cin/ exportacoes_rn_2013.pdf>. Acesso em: 30 jul. 2014.

FERRAZ, L. C. C. B. Gênero Pratylenchus-os nematóides das lesões radiculares. Revisão Anual de Patologia de Plantas, Passo Fundo, v. 7, n. 1, p. 157 $-195,1999$.

GONZAGA, V. Caracterização morfológica, morfométrica e multiplicação in vitro das seis espécies mais comuns de Pratylenchus Filipjev, 1936 que ocorrem no Brasil. 2006. 79 f. Tese (Doutorado em Agronomia: Área de concentração em Produção Vegetal) - Faculdade de Ciências Agrárias e Veterinárias - UNESP, Jaboticabal, 2006.

GOULART, A. M. C. Nematóides das Lesões Radiculares (Gênero Pratylenchus). AGROSOFT BRASIL. : [S.1.]. 2008. Disponível em:<www.agrosoft.org.br/agropag/103613.htlm>. Acesso em: 28 jul. 2014.

HANDOO, Z. A.; GOLDEN, A. M. A key and diagnostic compendium to the species of the genus Pratylenchus Filipjev, 1936 (lesion nematodes). Journal of Nematology, Hanover, v. 21, n. 2, p. 202 $-218,1989$.

HANDOO, Z. A.; CARTA, L. K.; SKANTAR, A. M. Morphological and molecular characterization of Pratylenchus arlingtoni $\mathrm{n}$. sp., $P$. convallariae and $P$. fallax (Nematoda: Pratylenchidae). Nematology, Leiden, v. 3, n. 6, p. 607-618, 2001.

HANDOO, Z. A.; CARTA, L. K.; SKANTAR, A. M. Taxonomy, Morphology and Phylogenetics of Coffee-Associated Root-Lesion Nematodes, Pratylenchus spp. In: SOUZA, R. M. (Ed.). PlantParasitic Nematodes of Coffee. Dordrecht: Springer Netherlands, 2008. cap. 3, p. 29-50.

BALMER, E.; PEREIRA, O. A. P. Doenças do milho. In: PATERNIANI, E.; VIEGAS, G. P. (Ed.). Melhoramento e produção do milho. Campinas: Fundação Cargill, 1987. v. 2, cap. 14, p. 595-634.

IBGE - Instituto Brasileiro de Geografia e Estatística. Produção Agrícola Municipal: Culturas Temporárias e Permanentes. Rio de Janeiro, RJ: IBGE, 2013. 102 p.

INSERRA, R.N. et al. Pratylenchus jaehni sp. n. from citrus in Brazil and its relationship with $P$. coffeae and P. loosi (Nematoda: Pratylenchidae). Nematology, Leiden, v. 3, n. 7, p. 653-665, 2001.

JENKINS, W.R. A rapid centrifugal-flotation technique for separating nematodes from soil. Plant Dis- ease Reporter, St. Paul, v. 48, n. 41, p. 692, 1964.

KARSSEN, G.; WAEYENBERGE, L.; MOENS, M. Pratylenchulus brzeskii sp. nov. (Nematoda: Pratylenchidae), a root-lesion nematode from european coastal dunes. Annales Zoologici, Warszawa, v. 50, n. 2, p. 255-261, 2000.

LIRA, V. L. Caracterização morfométrica e molecular de populações de Pratylenchus coffeae e reações de leguminosas e gramíneas ao parasitismo. 2013. 59 f. Dissertação (Mestrado: Área de concentração em Saúde Humana e Meio Ambiente) - Universidade Federal de Pernambuco - CAV, Vitória de Santo Antão, 2013.

LIRA, V. L. et al. Análises morfométrica e molecular de isolados de Pratylenchus coffeae ocorrentes no estado de Pernambuco, Brasil, em inhame. Nematropica, Arkansas, v. 44, n. 2, p. 152- 165, 2014.

MAI, W. F.; MULLIN P. G. Plant-Parasitic Nematodes: A Pictorial Key to Genera . 5 ed. New York: Cornell Univ. Press, 1996. 277 p.

MIZUKUBO, T.; SUGIMURA, K.; UESUGI, K. A new species of the genus Pratylenchus from chrysanthemum in Kyushu, western Japan (Nematoda: Pratylenchidae). Japanese Journal of Nematology, Hanover, v. 37, n. 2, p. 63-74, 2007.

MOURA, R. M.; TORRES, G. R. C. Os fitonematóides na fruticultura irrigada do nordeste: desafio a ser enfrentado. Anais da Academia Pernambucana de Ciência Agronômica, Recife, v. 1, s/n, p. 40-44, 2004.

PALOMARES-RIUS, J. E. et al. Description of Pratylenchus hispaniensis n. sp. from Spain and considerations on the phylogenetic relationship among selected genera in the Family Pratylenchidae. Nematology, Leiden, v. 12, n. 3, p. 429-451, 2010.

PENA, E. DE LA et al. Description of Pratylenchus dunensis sp. n. (Nematoda: Pratylenchidae), a rootlesion nematode associated with the dune grass $\mathrm{Am}$ mophila arenaria (L.) Link. Nematology, Leiden, v. 8, n. 1, p. 79-88, 2006.

RIO DE JANEIRO (Estado). Resolução SEAPEC No 69 DE 26/03/2015. Classifica o nematóide do café, Meloidogyne incognita, como praga prioritária e estabelece medidas para o controle da praga em cafezais, e dá outras providências. Diário Oficial do Estado, Rio de Janeiro, 31 mar. 2015. Disponível em:< http://www.legisweb.com.br/legislacao/? $\mathrm{id}=282562>$. Acesso em: 30 jul. 2015.

ROMAN, J.; HIRSCHMANN, H. Morphology and morphometrics of six species of Pratylenchus. Jour- 
nal of Nematology, Hanover, v. 1, n. 4, p. 363-386. 1969.

SIQUEIRA, K. M. S. et al. Detecção de Meloidogyne mayaguensis em goiabeira e mamoeiro no estado de Goiás, usando marcadores moleculares. Tropical Plant Pathology, Brasília, v. 34, n. 4, p. 256-260. 2009.

THORNE, G. Principles of Nematology. 1. ed. New York, 1961. $553 \mathrm{p}$.

TORRES, G. R.C. et al. Pratylenchus brachyurus em Cucumis melo no Brasil. Fitopatologia Brasileira, Brasília, v. 29, n. 6, p. 668-669. 2004b.

TORRES, G .R. C. et al. Meloidogyne mayaguensis em Psidium guajava no Rio Grande do Norte. Fitopatologia Brasileira, Brasília, v. 29, n. 5, p. 570. 2004a.

TORRES, G. R. C. et al. Hemicycliophora catarinensis associada à rizosfera de mudas de goiabeira no Nordeste. Nematologia Brasileira, Brasília, v. 29. n. 2. p. 303-305. 2005 b.

TORRES, G. R. C. et al. Ocorrência de Meloidogyne mayaguensis em goiabeira no Estado do Ceará. Nematologia Brasileira, Brasília, v. 29, n. 1, p. 105 107. 2005a.

TORRES, G. R. C. et al. Aspectos Ecológicos de Comunidade de Nematóides Associada a Cultivo de Cucumis melo no Rio Grande do Norte. Nematologia Brasileira, Brasília, v. 30, n. 1, p. 1-9. 2006.

TORRES, G. R. C.et al. Meloidogyne mayaguensis: novos assinalamentos no Rio Grande do Norte associados à goiabeira. Revista Caatinga, Mossoró, v. 20. n. 2. p. 106-112. 2007.

TROCCOLI, A. et al. Morphological and molecular characterization of Pratylenchus lentis n. sp. (Nematoda: Pratylenchidae) from Sicily. Journal of Nematology, Hanover, v. 40, n. 3, p.190-196, 2008. 